\title{
Repeatability of short-duration transient visual evoked potentials in normal subjects
}

\author{
Celso Tello • Carlos Gustavo V. De Moraes • \\ Tiago S. Prata $\cdot$ Peter Derr $\cdot$ Jayson Patel $\cdot$ \\ John Siegfried $\cdot$ Jeffrey M. Liebmann $\cdot$ Robert Ritch
}

Received: 23 March 2009/Accepted: 7 January 2010/Published online: 29 January 2010

(C) The Author(s) 2010. This article is published with open access at Springerlink.com

\begin{abstract}
To evaluate the within-session and intersession repeatability of a new, short-duration transient visual evoked potential (SD-tVEP) device on normal individuals, we tested 30 normal subjects (20/ 20 visual acuity, normal 24-2 SITA Standard VF) with SD-tVEP. Ten of these subjects had their tests
\end{abstract}

Presented in part at the Annual Meeting of the Association for Research in Vision and Ophthalmology, Fort Lauderdale, FL, USA, May 3-8, 2009.

C. Tello $(\bowtie)$. C. G. V. De Moraes .

T. S. Prata · J. M. Liebmann · R. Ritch

Department of Ophthalmology, Einhorn Clinical Research

Center, New York Eye and Ear Infirmary, 310 East 14th

Street, New York, NY 10003, USA

e-mail: doctorcelsotello@yahoo.com

C. Tello $\cdot$ R. Ritch

Department of Ophthalmology, The New York

Medical College, Valhalla, NY, USA

P. Derr · J. Patel

Diopsys, Inc., Pine Brook, NJ, USA

J. Siegfried

Pennsylvania College of Optometry at Salus University, Elkins Park, PA, USA

\section{J. M. Liebmann}

Manhattan Eye, Ear and Throat Hospital,

New York, NY, USA

J. M. Liebmann

New York University School of Medicine,

New York, NY, USA repeated within 1-2 months from the initial visit. Synchronized single-channel EEG was recorded using a modified Diopsys Enfant ${ }^{\mathrm{TM}}$ System (Diopsys, Inc., Pine Brook, New Jersey, USA). A checkerboard stimulus was modulated at two reversals per second. Two different contrasts of checkerboard reversal patterns were used: $85 \%$ Michelson contrast with a mean luminance of $66.25 \mathrm{~cd} / \mathrm{m}^{2}$ and $10 \%$ Michelson contrast with a mean luminance of $112 \mathrm{~cd} / \mathrm{m}^{2}$. Each test lasted $20 \mathrm{~s}$. Both eyes, independently and together, were tested 10 times (5 times at each contrast level). The following information was identified from the filtered N75-P100-N135 complex: N75 amplitude, N75 latency, P100 amplitude, P100 latency, and Delta Amplitude (N75-P100). The median values for each eye's five SD-tVEP parameters were calculated and grouped into two data sets based on contrast level. Mean age was $27.3 \pm 5.2$ years. For OD only, the median $(95 \%$ confidence intervals) of Delta Amplitude (N75-P100) amplitudes at $10 \%$ and $85 \%$ contrast were $4.6 \mathrm{uV}$ (4.1-5.9) and 7.1 uV (5.15-9.31). The median P100 latencies were $115.2 \mathrm{~ms}(112.0-117.7)$ and $104.0 \mathrm{~ms}$ (99.9-106.0). There was little within-session variability for any of these parameters. Intraclass correlation coefficients ranged between 0.64 and 0.98 , and within subject coefficients of variation were $3-5 \%$ (P100 latency) and 15-30\% (Delta Amplitude (N75P100) amplitude). Bland-Altman plots showed good agreement between the first and fifth test sessions (85\% contrast Delta Amplitude (N75-P100) delta 
amplitude, mean difference, $0.48 \mathrm{mV}, 95 \% \mathrm{CI}$, $-0.18-1.12 ; 85 \%$ contrast P100 latency delay, $-0.82 \mathrm{~ms}, 95 \% \mathrm{CI},-3.12-1.46 ; 10 \%$ contrast Delta Amplitude (N75-P100) amplitude, $0.58 \mathrm{mV}, 95 \% \mathrm{CI}$, $-0.27-1.45 ; 10 \%$ contrast P100 latency delay, $-2.05 \mathrm{mV}, 95 \% \mathrm{CI},-5.12-1.01)$. The inter-eye correlation and agreement were significant for both SD-tVEP amplitude and P100 latency measurements. For the subset of eyes in which the inter-session repeatability was tested, the intraclass correlation coefficients ranged between 0.71 and 0.86 with good agreement shown on Bland-Altman plots. Shortduration transient VEP technology showed good within-session, inter-session repeatability, and good inter-eye correlation and agreement.

Keywords Visual evoked potential . Electrophysiology $\cdot$ Repeatability

\section{Introduction}

The conventional pattern-reversal visual evoked potential (VEP) technique is used as an objective method of evaluating the visual pathway. Electrodes placed in standardized positions detect electrical signals generated by the visual cortex while the patient fixates at the center of a phase reversing checkerboard pattern. Since the VEP technique evaluates the final output of the entire visual system, it may be potentially useful for the detection of any type of visual abnormality [1]. However, problems such as positioning of electrodes, limited repeatability, subjective analysis of the waveforms, and long test duration have limited its use in clinical practice. Improvements on the original technique that could eliminate some or all of these problems have the potential to increase the clinical applicability of VEP as a screening tool for a range of eye diseases and visual conditions.

The Diopsys Enfant ${ }^{\mathrm{TM}}$ system (Diopsys, Inc., Pine Brook, New Jersey, USA) is a VEP technology that has been used as a screening tool for detecting amblyopia and other visual deficits in young children [2]. We modified this system in order to decrease test duration by means of a more efficient signal acquisition technique followed by a post-processing algorithm that provides less subjectivity in waveform assessment. Such improvements could possibly result in low test-retest repeatability that could compromise its clinical usefulness. We aimed to evaluate the within-session and inter-session repeatability of this modified VEP technique in normal individuals.

\section{Methods}

The study was approved by the Institutional Review Board of New York Eye and Ear Infirmary and The Pennsylvania College of Optometry of Salus University and informed consent was obtained from all participants.

Thirty normal individuals were prospectively enrolled. Subjects underwent complete ophthalmologic examination, including slit-lamp biomicroscopy, dilated fundus examination and visual field testing (24-2 SITA-Standard strategy, Carl Zeiss Meditec, Inc., Dublin, CA). All individuals had 20/ 20 best corrected visual acuity, normal biomicroscopic examination and normal field tests.

\section{Apparatus}

Transient VEPs (tVEP) were generated using a Diopsys Enfant ${ }^{\mathrm{TM}}$ System (Diopsys, Inc., Pine Brook, New Jersey, USA), with software modifications. Because the test duration was shortened considerably by the modifications to the system, we have termed this testing paradigm short-duration tVEP (SD-tVEP).

The stimulus was presented on a gamma-corrected Phillips 170B7 17-inch LCD monitor, running at $75 \mathrm{~Hz}$. Luminance output over time was verified using a luminance meter (MavoSpot 2 USB, Gossen Gmb, Nuremberg, Germany). The monitor was set to operate in the linear luminance region with less than 4\% measured residual flicker. Gold cup electrodes (Grass Model F-E5GH, Astro-Med, Inc., West Warwick, RI) $(10 \mathrm{~mm})$ and commercially available skin preparation and EEG paste were used for recording of the SD-tVEP. Synchronized single-channel SD-tVEP waves were recorded and generated a time series of 240 data points per analysis window.

\section{Stimulus}

The circular black/white checkerboard pattern reversal stimulus had a diameter of $22 \mathrm{~cm}$ with a red 
circular outline used as fixation target. The diameter of this target was $1 \mathrm{~cm}$ with a thickness of $1 \mathrm{~mm}$. The check size was $28.87 \mathrm{~min}$ of arc. Two pattern contrasts were used in the study, based on previous studies that suggested that differential contrast stimulation could affect the VEP waveforms [3, 4]. The first pattern had white checks of $122.9 \mathrm{~cd} / \mathrm{m}^{2}$ and black checks of $101.1 \mathrm{~cd} / \mathrm{m}^{2}$ resulting in a Michelson contrast of $10 \%$ and mean luminance of $112 \mathrm{~cd} / \mathrm{m}^{2}$. The second pattern had white checks of $122.9 \mathrm{~cd} / \mathrm{m}^{2}$ and black checks of $9.59 \mathrm{~cd} / \mathrm{m}^{2}$ resulting in a Michelson contrast of $85 \%$ and mean luminance of $66.25 \mathrm{~cd} / \mathrm{m}^{2}$. The two patterns will be referred to as $10 \%$ contrast and $85 \%$ contrast. In all cases, the display was viewed through natural pupils with optimal refractive correction in place. The viewing distance was set to 1 meter, yielding a total display viewing angle of 12.54 degrees.

During a recording session, the luminance of each stimulus check was temporally modulated in counter phase at $1 \mathrm{~Hz}$ (two reversals per second). The 10 and $85 \%$ contrast stimuli were presented in a sequence of $\mathrm{OS}$, OD, and $\mathrm{OU}$ for a minimum of 40 pattern reversals each. If a motion or blink artifact was detected, that epoch was ignored and repeated automatically. During monocular testing, the fellow eye was covered. The sequence was repeated five times with a 3-min break between each sequence. The five sequences were first done at $10 \%$ contrast and then repeated at the $85 \%$ contrast level.

Since an LCD display will cause apparent (artificial) delay in the P100 latency due to the input lag of the monitor [5], software modifications were made to compensate for the lag. This lag is caused by the finite time the LCD display takes to process the input video signal and display the pattern. This inherent delay of the LCD display was calculated using an optical feedback circuit. The standard filter/amplifier circuit and electrodes that would normally record the SD-tVEP was replaced with a photo detector circuit. The photo detector circuit generated a distinctive signal every time the pattern on the monitor was phase reversed. This signal was compared to the monitor's input signal, and the length of the delay was calculated.

Five normal subjects were tested on both an LCD and CRT monitor to test for the effects of the asymmetrical luminance onset/offsets (flicker) of the LCD monitor. A Phillips 170B7 LCD monitor and a
Mitsubishi SpectraView 7000 were used for the comparison study. Testing only OD, 15 tests $(20 \mathrm{~s}$ test time, 2 reversals per second, $85 \%$ contrast) were performed with a 10-min break between monitors. The P100 latency and delta amplitude were compared between the monitors, and the results analyzed using a paired sample $t$ test. For all patients tested, there was no significant difference between the two groups (alpha $=0.05)$ for P100 latency and delta amplitude.

\section{Recording procedures}

One EEG channel was recorded using gold cup electrodes. The electrodes were placed $4-\mathrm{cm}$ above the inion (active) and on the frontal tuberosity centered on the midline (reference). While this deviates from the International Society for Clinical Electrophysiology of Vision (ISCEV) protocol of $10 \%$ of the inion-nasion distance, an approximation of $4 \mathrm{~cm}$ was used to decrease overall test preparation time. The left side of the forehead just in front of the temple served as ground.

In preparation for recording, the skin at each electrode site was scrubbed with Nuprep (D.O. Weaver \& Co., Aurora, CO) on a cotton-tipped wooden swab. Electrodes were fixed in position with Ten 20 conductive paste (D.O. Weaver \& Co., Aurora, CO) and secured with a small gauze pad with conductive paste applied. Electrode impedance was maintained below 10k ohms in all cases and was usually below $5 \mathrm{k}$ ohms. Analog signals were amplified by $10^{4}$ (Diopsys Enfant Amp 100, Diopsys, Inc., Pine Brook, New Jersey, USA), band-pass filtered with cut-off frequencies of $0.5-100 \mathrm{~Hz}$ and sampled at $600 \mathrm{~Hz}$ using the Enfant System.

Data analysis

The stored SD-tVEP data for each subject was exported from the device's relational database to an external binary file to be processed by an external signal processing algorithm [6]. All subjects had their data imported into the signal processing algorithm. The data had the N75-P100-N135 complex temporal epoch identified by windowing and bilateral band pass filtering utilizing Derr's extraction method [6]. The low pass cutoff frequency was chosen to be $30 \mathrm{~Hz}$. The high pass frequency was set to $5.1 \mathrm{~Hz}$ so as to minimize low frequency drift. Reducing this 
drift enhances an automated method for isolating the N75-P100-N135 complex. The following information was identified from the filtered N75-P100-N135 complex: N75 amplitude, N75 latency, P100 amplitude, P100 latency, and Delta Amplitude (N75-P100) amplitude.

\section{Statistical analysis}

The mean $(\mu)$ and standard deviations (SD) for the five SD-tVEP, for each OD and OS at each contrast level were calculated, and their ratio $(\mathrm{SD} / \mu)$ defined the within-subject coefficient of variation $(\mathrm{Sw})$. The standard deviations for OD and OS were grouped into two data sets based on contrast level. Intraclass correlation coefficients (ICC) were calculated for the Delta Amplitude (N75-P100) amplitudes and P100 latency for one eye. To test the degree of inter-eye symmetry in the responses, we calculated the coefficients $(r)$ based on Pearson's correlation between the average values of OD and OS. Bland-Altman plots were created to evaluate the inter-eye agreement, as well as the agreement between the first and last t-VEP run for each eye at each contrast level. To test the inter-session repeatability, a subset of ten patients (one eye per patient) of this database had their tests repeated within 1-2 months of the initial visit. Data from day 1 and day 2 were compared. The results from $\mathrm{OU}$ runs were not included in the statistical analysis.

\section{Results}

Thirty normal subjects were enrolled. The total number of tests was 900 [30 patients $\times 3(\mathrm{OD}$, OS, OU) $\times 5$ (re-tests $) \times 2(10$ and $85 \%$ contrast $)]$. The mean age was $27.3 \pm 5.2$ years; $66 \%$ were men and $53 \%$ were of European ancestry. Figures 1 and 2 show an overlay of five consecutive tests of the right eye of one the subjects.

The median, first and third quartiles, and interquartile range (IQR) for each parameter are shown in Table 1 (A and B). Considering the N75-P100 amplitudes at $10 \%$ contrast, the median values for this population were $4.63,4.11$, and $5.55 \mathrm{uV}$ for OD, $\mathrm{OS}$, and OU, respectively. Using $85 \%$ contrast, the Delta Amplitude (N75-P100) amplitude median values were 7.13, 6.98, and 7.2 uV. The median P100

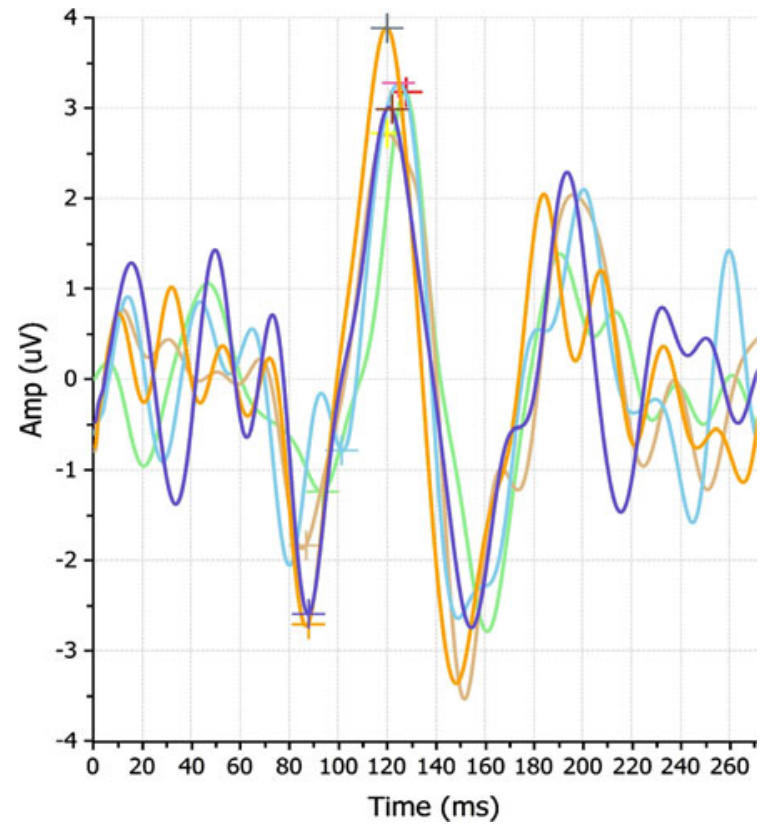

Fig. 1 An overlay of five OD 10\% contrast tests

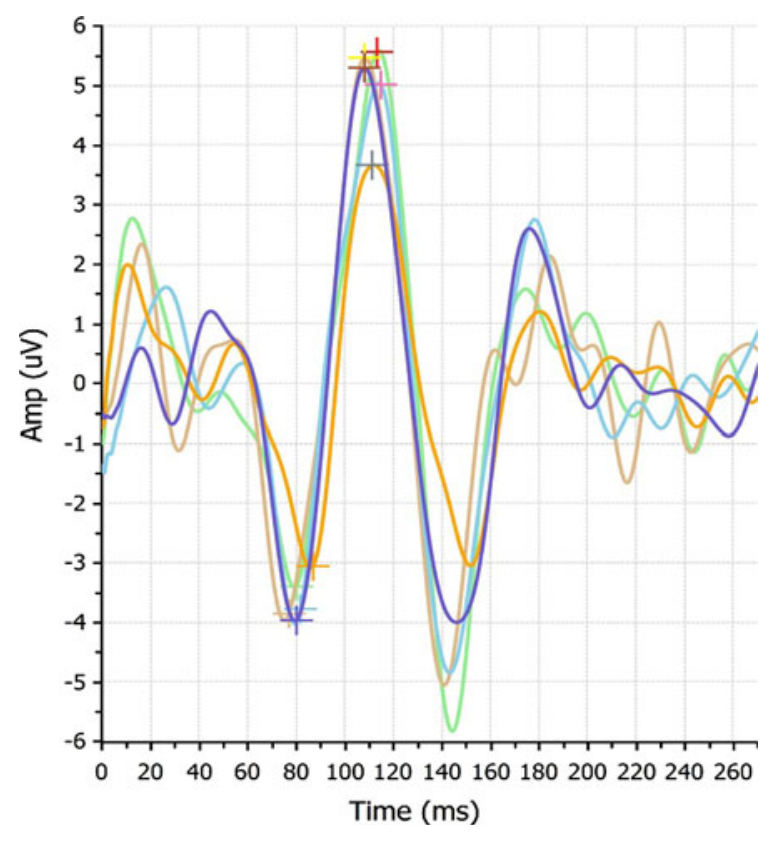

Fig. 2 An overlay of five OD $85 \%$ contrast tests

latencies at $10 \%$ contrast were $115.17,114.83$, and $109.33 \mathrm{~ms}$ for OD, OS, OU, respectively. At $85 \%$ contrast, the median P100 latencies were 99.52, 100.89 , and $98.50 \mathrm{~ms}$. Table 2 shows the repeatability indices for each parameter. The ICC values at 
Table 1 Summary of test-retest repeatability of different parameters using SD-tVEP

\begin{tabular}{|c|c|c|c|c|c|c|c|}
\hline & Minimum & $\begin{array}{l}\text { First } \\
\text { quartile }\end{array}$ & Median & $95 \% \mathrm{CI}$ & $\begin{array}{l}\text { Third } \\
\text { quartile }\end{array}$ & Maximum & IQR \\
\hline \multicolumn{8}{|l|}{ (A) $10 \%$ contrast } \\
\hline \multicolumn{8}{|l|}{ OD only } \\
\hline N75 Amp. (uV) & -2.73 & -1.25 & -0.95 & $(-1.07,-0.85)$ & -0.82 & -0.53 & 0.43 \\
\hline N75 Lat. (ms) & 64.00 & 74.94 & 79.67 & $(75.67,84.67)$ & 86.00 & 98.33 & 11.06 \\
\hline P100 Amp. (uV) & 2.05 & 2.76 & 3.65 & $(3.26,4.95)$ & 5.64 & 8.51 & 2.88 \\
\hline P100 Lat. (ms) & 105.67 & 110.28 & 115.17 & $(112.0,117.67)$ & 118.33 & 127.00 & 8.06 \\
\hline Delta Amp. (N75-P100) (uV) & 2.86 & 3.55 & 4.63 & $(4.12,5.89)$ & 6.91 & 10.99 & 3.36 \\
\hline \multicolumn{8}{|l|}{ OS only } \\
\hline N75 Amp. (uV) & -2.25 & -1.06 & -0.82 & $(-0.95,-0.72)$ & -0.71 & -0.49 & 0.35 \\
\hline N75 Lat. (ms) & 63.33 & 77.92 & 82.33 & $(79.67,85.00)$ & 88.14 & 97.67 & 10.22 \\
\hline P100 Amp. (uV) & 1.75 & 2.83 & 3.35 & $(2.93,3.86)$ & 4.39 & 10.70 & 1.56 \\
\hline P100 Lat. (ms) & 104.00 & 111.89 & 114.83 & $(113.00,116.67)$ & 119.67 & 124.33 & 7.78 \\
\hline Delta Amp. (N75-P100) (uV) & 2.29 & 3.61 & 4.11 & $(3.68,4.91)$ & 5.18 & 12.83 & 1.57 \\
\hline \multicolumn{8}{|l|}{ OU only } \\
\hline N75 Amp. (uV) & -2.21 & -1.15 & -1.02 & $(-1.12,-0.81)$ & -0.72 & -0.31 & 0.43 \\
\hline N75 Lat. (ms) & 57.00 & 70.92 & 76.67 & $(73.33,79.33)$ & 81.25 & 108.33 & 10.33 \\
\hline P100 Amp. (uV) & 1.79 & 3.60 & 4.60 & $(3.85,5.64)$ & 6.06 & 12.83 & 2.46 \\
\hline P100 Lat. (ms) & 95.67 & 104.33 & 109.33 & $(105.67,112.33)$ & 113.33 & 130.00 & 9.00 \\
\hline Delta Amp. (N75-P100) (uV) & 2.26 & 4.51 & 5.55 & $(4.68,6.67)$ & 7.22 & 15.02 & 2.71 \\
\hline \multicolumn{8}{|l|}{ (B) $85 \%$ contrast } \\
\hline \multicolumn{8}{|l|}{ OD only } \\
\hline N75 Amp. (uV) & -12.58 & -3.96 & -2.50 & $(-3.23,-1.78)$ & -1.67 & -0.68 & 2.30 \\
\hline N75 Lat. (ms) & 62.34 & 71.30 & 74.67 & $(73.32,77.33)$ & 77.44 & 83.66 & 6.14 \\
\hline P100 Amp. (uV) & 1.74 & 2.38 & 4.46 & $(2.88,5.86)$ & 6.70 & 13.69 & 4.32 \\
\hline P100 Lat. (ms) & 94.64 & 99.52 & 104.00 & $(99.96,106.00)$ & 107.05 & 111.34 & 7.53 \\
\hline Delta Amp. (N75-P100) (uV) & 2.64 & 4.63 & 7.13 & $(5.15,9.31)$ & 10.18 & 26.30 & 5.55 \\
\hline \multicolumn{8}{|l|}{ OS only } \\
\hline N75 Amp. (uV) & -13.88 & -4.19 & -2.57 & $(-3.24,-1.78)$ & -1.69 & -0.86 & 2.49 \\
\hline N75 Lat. (ms) & 69.99 & 75.22 & 78.00 & $(75.34,80.00)$ & 81.08 & 90.32 & 5.86 \\
\hline P100 Amp. (uV) & 1.75 & 2.41 & 4.75 & $(2.73,6.12)$ & 6.31 & 15.15 & 3.90 \\
\hline P100 Lat. (ms) & 96.30 & 100.89 & 103.99 & $(101.98,106.96)$ & 107.37 & 115.96 & 6.49 \\
\hline Delta Amp. (N75-P100) (uV) & 3.01 & 4.61 & 6.98 & $(5.07,9.67)$ & 10.03 & 29.03 & 5.42 \\
\hline \multicolumn{8}{|l|}{ OU only } \\
\hline N75 Amp. (uV) & -13.47 & -4.62 & -2.76 & $(-4.10,-2.18)$ & -2.06 & -1.30 & 2.56 \\
\hline N75 Lat. (ms) & 60.66 & 73.30 & 76.32 & $(74.96,78.00)$ & 79.14 & 88.03 & 5.84 \\
\hline P100 Amp. (uV) & 1.79 & 2.59 & 4.72 & $(2.99,6.24)$ & 6.57 & 12.02 & 3.97 \\
\hline P100 Lat. (ms) & 94.32 & 98.50 & 102.49 & $(98.66,105.66)$ & 107.33 & 115.16 & 8.83 \\
\hline Delta Amp. (N75-P100) (uV) & 3.55 & 4.77 & 7.21 & $(5.08,11.16)$ & 11.67 & 25.50 & 6.90 \\
\hline
\end{tabular}

$10 \%$ contrast P100 latency and Delta Amplitude (N75-P100) amplitudes were 0.64 and 0.92 , respectively. At $85 \%$ contrast, the values were 0.65 and 0.98 , respectively. The within-subject coefficients of variation at $10 \%$ contrast were 5 and $30 \%$, and at $85 \%$ they were 3 and $15 \%$, respectively. BlandAltman plots showed good agreement between the first and fifth test sessions. At $85 \%$ contrast, Delta 
Table 2 Intraclass correlation coefficients (ICC), standard deviation (SD), and within-subject coefficient of variation (Sw) of the tested parameters

\begin{tabular}{llllc}
\hline & ICC & $95 \%$ CI & SD & Sw (\%) \\
\hline 10\% P100 latency (ms) & 0.64 & $0.41-0.81$ & 5.5 & 5 \\
10\% N75-P100 & 0.92 & $0.86-0.95$ & 1.4 & 30 \\
$\quad$ amplitudes (uV) & & & & \\
85\% P100 latency (ms) & 0.65 & $0.42-0.81$ & 4.0 & 3 \\
85\% N75-P100 & 0.98 & $0.97-0.99$ & 1.2 & 15 \\
$\quad$ amplitudes (uV) & & & & \\
\hline
\end{tabular}

Amplitude (N75-P100) mean difference was $0.48 \mathrm{uV}$, with $95 \%$ confidence interval $(\mathrm{CI})$ between -0.18 and 1.12; for P100 latency, the mean was $-0.82 \mathrm{~ms}$, 95\% CI, -3.12-1.46. At 10\% contrast, Delta Amplitude (N75-P100) mean difference was $0.58 \mathrm{uV}, 95 \%$ CI, -0.27-1.45; and for P100 latency delay, the mean difference was $-2.05 \mathrm{uV}, 95 \% \mathrm{CI},-5.12-1.01$ (Fig. 3a-d).

For the $85 \%$ contrast tests, there was a strong and significant correlation of both delta amplitudes and P100 latencies between fellow eyes $(r=0.93$ and $r=0.73, \quad P<0.01$, respectively). For the $10 \%$ contrast tests, the correlation was also strong and significant $(r=0.89$ and $r=0.62, P<0.01$, respectively). The Bland-Altman plots are presented in Fig. 4a-d. There was a good overall agreement between fellow eyes for both Delta Amplitude (N75-P100) amplitudes and P100 latencies at 10 and $85 \%$ contrast. The mean difference between fellow eyes ranges from 0.1 to $0.3 \mathrm{uV}$ for amplitudes and $0.5 \mathrm{~ms}$ for latency.

Due to the length of testing protocol in this study, there was a need to investigate the effects of patient fatigue. All patient data from individual trials were pooled together and analyzed for trends of decreasing amplitude and/or increasing latency. The results indicated minimal effects of fatigue. The ranges for the median values of the amplitude and latency at $10 \%$ were (4.14-5.47 uV) and (114.17-117.50 ms), respectively. The $85 \%$ values were $(6.60-7.87 \mathrm{uV})$ and (103.30 and $105.00 \mathrm{~ms}$ ).

The inter-session repeatability $\triangle \mathrm{ICC}$ values for $10 \%$ latency and amplitudes were $0.86(0.46-0.96)$ and 0.85 (0.40-0.96), respectively. For $85 \%$ latency and amplitude, the values were $0.71 \quad(95 \%$ $\mathrm{CI}=0.15-0.92)$ and $0.81(0.23-0.95)$, respectively. Bland-Altman plots comparing the results of days 1 and 2 are shown in Fig. 5a-d.
Fig. 3 Bland-Altman plots showing the agreement between mean values of OD and OS. a $85 \%$ contrast Delta Amp. (N75-P100) $(\mathrm{uV})$, b $10 \%$ contrast Delta Amplitude (N75-P100) (uV), c $85 \%$ contrast P100 latency (ms), d 10\% P100 contrast latency (ms)
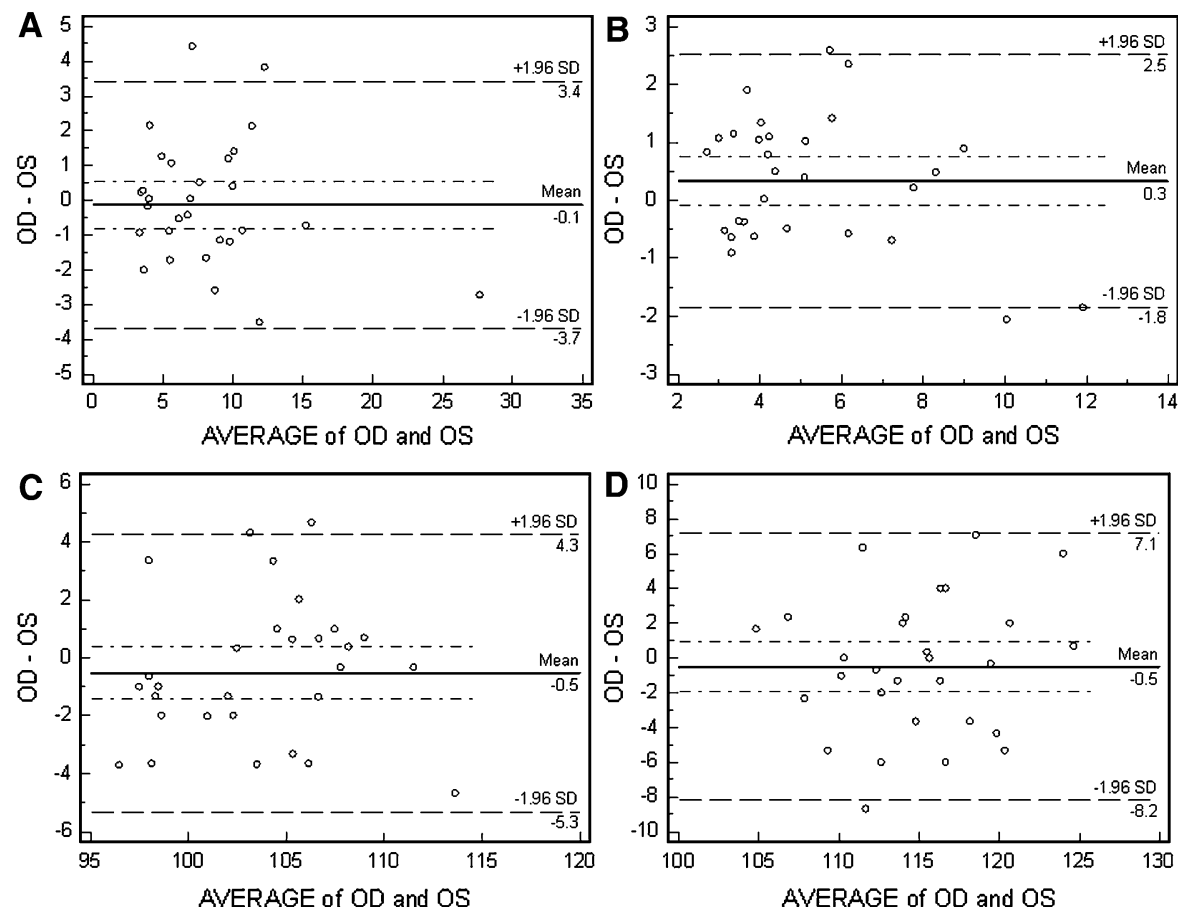

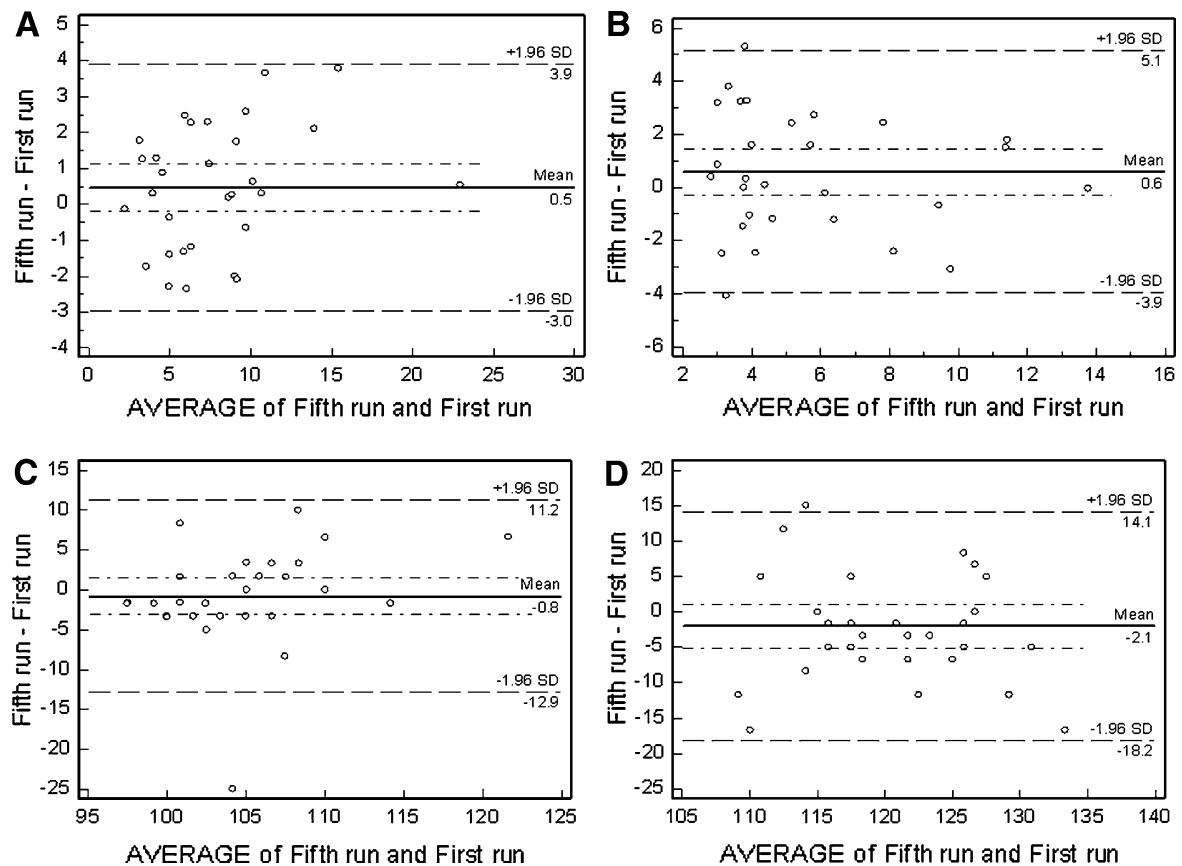

Fig. 4 Bland-Altman plots showing the within-session agreement between first and fifth run of the short duration t-VEP. a 85\% contrast Delta Amp. (uV), b $10 \%$ contrast Delta

\section{Discussion}

We evaluated the within-session and inter-session repeatability of a new SD-tVEP technique in normal subjects. Using 10 and $85 \%$ contrast, the responses to checkerboard contrast reversal stimuli showed good repeatability. There was a strong agreement between the first and fifth run of the subjects, as well as between two separate visits. In addition, we found a strong and significant inter-eye agreement and correlation among subjects. Such results were obtained during a test run time of $20 \mathrm{~s}$ per eye (40 reversals) and still allowed straightforward determination of the P100 peak latencies and amplitudes.

The within-subject repeatability of visual evoked potentials has been investigated in different populations using various modalities of the technique [7-11]. Each technique has its own characteristics, and comparisons should be made within the same technology. Also, the way repeatability is tested and measured may vary according to the study. Using multifocal VEP technique, Chen et al. [10] found strong correlations (Pearson's $r=0.88-0.92$ ) of amplitudes obtained between two sessions in the same day, and SD values were 1.1-1.5 (measured in

Amplitude (N75-P100) (uV), c 85\% contrast P100 latency (ms), d $10 \%$ contrast P100 latency (ms)

root-mean square). Lauritzen et al. [11] found a mean coefficient of variation of swept visual evoked potential (SWEEP-VEP) amplitudes of $16 \pm 7 \%$ among seven healthy adults at $80 \%$ contrast. Future studies ought to replicate our findings using the same technique.

Derr et al. [Derr P, Tello C, De Moraes CG, Patel J, Prata TS, Sigfried J, Liebmann JM, Ritch R (2009) Fast transient VEP parameter identification algorithm for evaluating glaucoma eyes. Invest Ophthalmol Vis Sci 50: E-Abstract 5886] showed an improved performance of the herein described algorithm in identifying the N75-P100-N135 complex both in normal subjects $(n=60)$ and glaucomatous eyes $(n=84)$. Compared to visual inspection of the raw VEP waveforms, the method allowed better discrimination between glaucomatous and healthy eyes. This information combined with the findings of the present study support a potential applicability of this technique as an adjunctive technology in eye care, similarly to what has been reported using other VEP technologies [12-18].

Even though we did not aim to compare patient performance at each contrast level, we found lower amplitudes and increased latencies using 10\% 

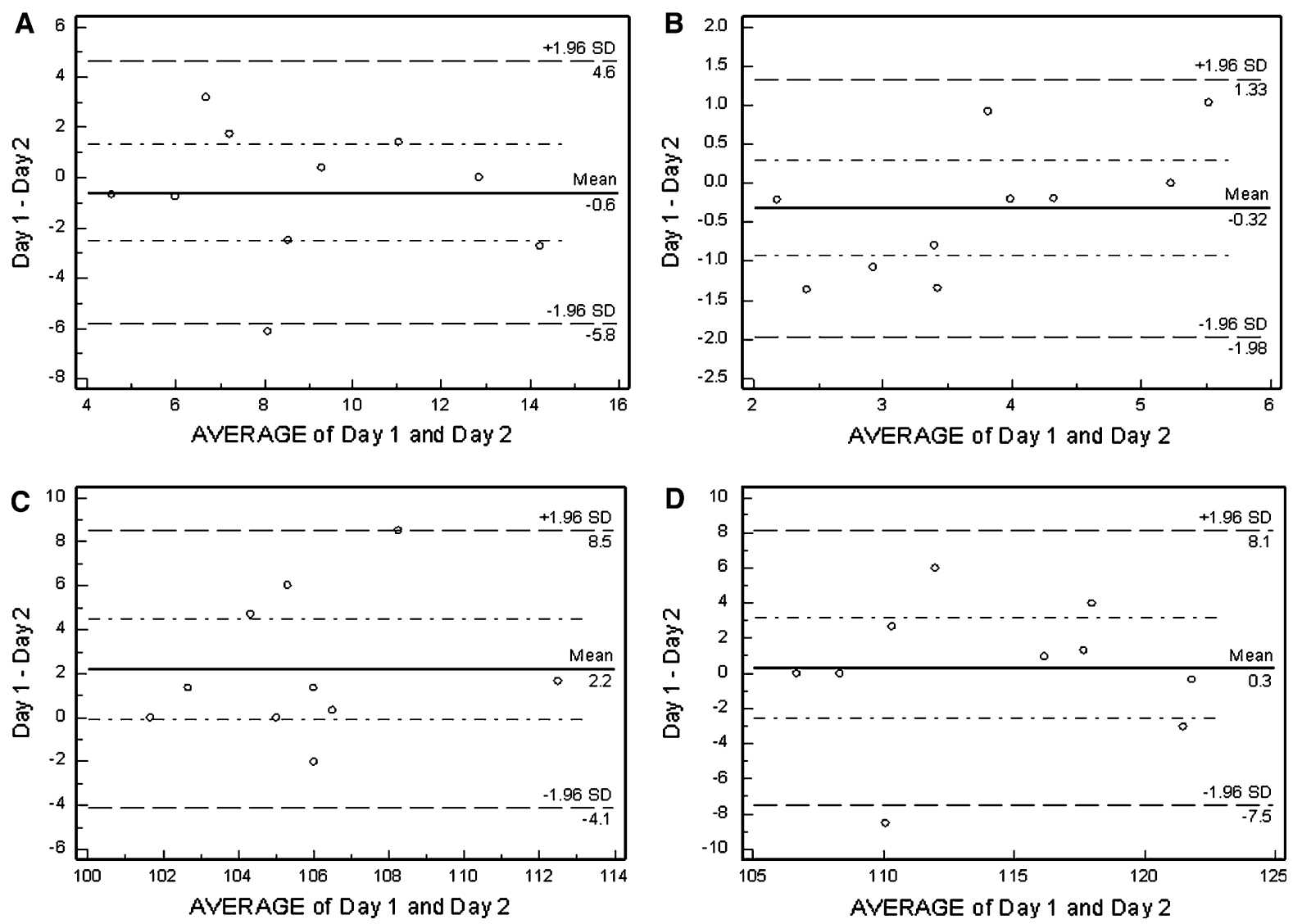

Fig. 5 Bland-Altman plots showing the inter-session agreement between days 1 and 2 of the short duration t-VEP. a $85 \%$ contrast Delta Amp. (N75-P100) (uV), b 10\% contrast Delta

contrast than $85 \%$ contrast. Some of these differences may have been due to the results of the change in luminance between contrast levels. Moreover, at $85 \%$ we found narrower confidence intervals both for the median values and within-session variation than at $10 \%$ contrast. The influence of the stimulus contrast on the VEP parameters was first observed by Duwaer and Spekreijse [19] in patients with multiple sclerosis: with lower contrast, the amplitude becomes smaller and the latency may increase. Changes in VEP responses associated with luminance contrast stimuli have also been observed in other diseases such as glaucoma [3, 4]. Using different contrast stimuli in patients could be employed in the future to try to evaluate selective visual dysfunction in various ocular diseases. This device's hardware would require modifications to the display as to maintain constant luminance for each contrast level.

Amplitude (N75-P100) (uV), c 85\% contrast P100 latency (ms), d 10\% contrast P100 latency (ms)

It is important to note that our sample was a relatively young population and patient age should be taken into account when interpreting VEP waveforms [20-22]. Shaw and Cant [20] showed that the amplitudes are highest in childhood, decrease up to the fourth decade, increase again, but decrease once more after the sixth decade. The latency may also increase in later life. Thus, the normative values found in our study should be interpreted cautiously when compared with older individuals. This factor notwithstanding, the new device showed good withinsession and inter-session repeatability in a young healthy sample.

We found a significant inter-eye correlation and agreement with this device. Interocular variation should be more precise when used to detect abnormalities than a comparison with a group of normal controls [23], especially in asymmetric diseases (e.g.: amblyopia, glaucoma, age-related macular 
degeneration, optic neuritis). By comparing the differences in amplitude or latency between fellow eyes, one can correlate with other clinical findings (such as fundus examination, intraocular pressure, and refractive error) and increase the likelihood of detecting eye diseases which are often asymmetric. This may be particularly helpful in subtle or early stage conditions in which the clinical examination provides insufficient information to establish a diagnosis based upon the assessment of a single eye alone compared to a normative database. The good intereye agreement and correlation found in our study could therefore be further investigated regarding the ability of the SD-tVEP in detecting early asymmetric eye diseases. Such potential use has been successfully demonstrated in patients with glaucoma using multifocal technology, for instance [24].

The observation that this test also presented good inter-session repeatability may have significant implications. It is known that the simple act of removing the electrodes and immediately repositioning them could cause variation in the measured electrical responses. Such inter-session variability could be a limitation for its use in certain conditions, especially for those requiring longitudinal evaluation of disease progression and/or response to treatment. As demonstrated in our results, there was a reasonable intersession agreement when patients were tested on different days. With a larger and age-matched database, this could be used for follow-up of chronic diseases such as diabetic maculopathy and glaucoma, for instance.

The study's objective was to determine the withinsession and inter-session repeatability of the proposed apparatus, using a specified screening protocol in conjunction with post-processing the signal-averaged VEP with a test time of $20 \mathrm{~s}$. This short-duration test time was not an issue for the normal population, but might not be adequate for all populations. Methods analyzing the signal to noise ratio with a stop criterion might prove useful in patients with ophthalmological disease.

One limitation of the conventional VEP technique is its high variability between individuals. The anatomy of the striate cortex (primary visual cortex or V1 area) varies greatly among individuals [25-28] and could also be influenced by the variation of extrastriate areas of the cortex [29-31]. There is also variation of the recorded responses dependent on patients' attention and accommodation [32], which we tried to minimize using a central target for fixation.

The term "short duration transient VEP" (SDtVEP) was named so as to reflect the time required to perform a testing protocol on a patient. It is important to note that the time to perform the test includes acquiring forty $500 \mathrm{~ms}$ epochs of tVEP data and performing objective analysis on the resulting signalaveraged response. The analysis, based on the Derr algorithm [6] automatically extracts a reproducible N75-P100-N135 complex from $20 \mathrm{~s}$ of VEP data and calculates all parametric values. As a result of the objective analysis, the protocol or a modified protocol may allow for VEP technology to become a viable screening device. It should also be noted that the devices' proprietary synchronization of pattern presentation and data collection adds to a cleaner signal. Combining the system's ability to lift the VEP signal out of the noise in 40 epochs, and the automatic extraction algorithm being able to detect this signal allows for a 20 -s run.

In summary, our study showed that a new VEP technique with shorter test duration and simplified waveform extraction showed good within-session and inter-session repeatability, as well as strong inter-eye correlation. Further investigation to determine its usefulness as a screening tool for abnormalities of the visual pathways is warranted.

Acknowledgments Celso Tello, Robert Ritch, Jeffrey Liebmann, and John Siegfried serve as consultants or have performed contract research for Diopsys, Inc. Peter Derr and Jayson Patel are employees of Diopsys, Inc.

Open Access This article is distributed under the terms of the Creative Commons Attribution Noncommercial License which permits any noncommercial use, distribution, and reproduction in any medium, provided the original author(s) and source are credited.

\section{References}

1. Odom JV, Bach M, Barber C, Brigell M, Marmor MF, Tormene AP, Holder GE, Vaegan (2004) Visual evoked potentials standard. Doc Ophthalmol 108:115-123

2. Simon JW, Siegfried JB, Mills MD, Calhoun JH, Gurland JE (2004) A new visual evoked potential system for vision screening in infants and young children. J AAPOS 8:549-554

3. Porciatti V, Di Bartolo E, Nardi N, Fiorentini A (1997) Responses to chromatic and luminance contrast in 
glaucoma: a psychophysical and electrophysiological study. Vis Res 37:1975-1987

4. Hart WM Jr, Silverman SE, Trick GL, Nesher R, Gordon MO (1990) Glaucomatous visual field damage: luminance and color-contrast sensitivities. Invest Ophthalmol Vis Sci 31:359-367

5. Husain A, Hayes S, Young M, Shah D (2009) Visual evoked potentials with CRT and LCD monitors: when newer is not better. Neurology 72:162-164

6. Derr PH, Meyer AU, Haupt EJ, Brigell MG (2002) Extraction and modeling of the oscillatory potential: signal conditioning to obtain minimally corrupted oscillatory potentials. Doc Ophthalmol 104:37-55

7. Towle VL, Moskowitz A, Sokol S, Schwartz B (1983) The visual evoked potential in glaucoma and ocular hypertension: effects of check size, field size, and stimulation rate. Invest Ophthalmol Vis Sci 24:175-183

8. Schellberg D, Gasser T, Köhler W (1987) The intra-individual reproducibility of flash-evoked potentials in a sample of children. Int J Psychophysiol 5:135-143

9. Joost W, Bach M (1990) Variability of the steady-state visually evoked potential: interindividual variance and intraindividual reproducibility of spatial frequency tuning. Doc Ophthalmol 75:59-66

10. Chen CS, Hood DC, Zhang X, Karam EZ, Liebmann JM, Ritch R, Thienprasiddhi P, Greenstein VC (2003) Repeat reliability of the multifocal visual evoked potential in normal and glaucomatous eyes. J Glaucoma 12:399-408

11. Lauritzen L, Jørgensen MH, Michaelsen KF (2004) Testretest reliability of swept visual evoked potential measurements of infant visual acuity and contrast sensitivity. Pediatr Res 55:701-708

12. Galloway NR, Tolia J, Barber C (1986) The pattern evoked response in disorders of the optic nerve. Doc Ophthalmol 63:31-36

13. Bray LS, Mitchell KW, Howe JW et al (1992) Visual function in glaucoma: a comparative evaluation of computerized static perimetry and the pattern visual evoked potential. Clin Vis Sci 7:21-29

14. Watts MT, Good PA, O'Neill EC (1989) The flash stimulated VEP in the diagnosis of glaucoma. Eye 3:732-737

15. Grover LK, Hood DC, Ghadiali Q et al (2008) A comparison of multifocal and conventional visual evoked potential techniques in patients with optic neuritis/multiple sclerosis. Doc Ophthalmol 117:121-128

16. Grippo TM, Hood DC, Kanadani FN et al (2006) A comparison between multifocal and conventional VEP latency changes secondary to glaucomatous damage. Invest Ophthalmol Vis Sci 47:5331-5536

17. Graham SL, Klistorner AI, Goldberg I (2005) Clinical application of objective perimetry using multifocal visual evoked potentials in glaucoma practice. Arch Ophthalmol 123:729-739

18. Hood DC, Zhang X (2000) Multifocal ERG and VEP responses and visual fields: comparing disease-related changes. Doc Ophthalmol 100:115-137

19. Duwaer AL, Spekreijse H (1978) Latency of luminance and contrast evoked potentials in multiple sclerosis patients. Electroencephalogr Clin Neurophysiol 45:244-258

20. Shaw NA, Cant BR (1981) Age-dependent changes in the amplitude of the pattern visual evoked potential. Electroencephalogr Clin Neurophysiol 51:671-673

21. Dustman RE, Snyder EW, Schlehuber CJ (1981) Life-span alterations in visually evoked potentials and inhibitory function. Neurobiol Aging 2:187-192

22. Sokol S, Moskowitz A, Towle VL (1981) Age-related changes in the latency of the visual evoked potential: influence of check size. Electroencephalogr Clin Neurophysiol 51:559-562

23. Brigell M, Bach M, Barber C et al (1998) Guidelines for calibration of stimulus and recording parameters used in clinical electrophysiology of vision. Calibration Standard Committee of the International Society for Clinical Electrophysiology of Vision (ISCEV). Doc Ophthalmol 95:1-14

24. Hood DC, Greenstein VC (2003) Multifocal VEP and ganglion cell damage: applications and limitations for the study of glaucoma. Prog Retin Eye Res 22:201-251

25. Brindley GS (1972) The variability of the human striate cortex. J Physiol 225:1-3

26. Stensaas SS, Eddington DK, Dobelle WH (1974) The topography and variability of the primary visual cortex in man. J Neurosurg 40:747-755

27. Rademacher J, Caviness VS Jr, Steinmetz H, Galaburda AM (1993) Topographical variation of the human primary cortices: implications for neuroimaging, brain mapping, and neurobiology. Cereb Cortex 3:313-329

28. Aine CJ, Supek S, George JS et al (1996) Retinotopic organization of human visual cortex: departures from the classical model. Cereb Cortex 6:354-361

29. Jeffreys DA (1970) Striate and extra-striate origins of pattern- related visual evoked potential (VEP) components. J Physiol 211:29-30

30. Srebro R (1985) Localization of visually evoked cortical activity in humans. J Physiol 360:233-246

31. Ossenblok P, Spekreijse H (1991) The extrastriate generators of the EP to checkerboard onset: a source localization approach. Electroencephalogr Clin Neurophysiol 80:181193

32. Mezer E, Bahir Y, Leibu R, Perlman I (2004) Effect of defocusing and of distracted attention upon recordings of the visual evoked potential. Doc Ophthalmol 109: 229-238 